Mathematical Modelling and Analysis

Volume 20 Number 1, January 2015, 76-93

http://dx.doi.org/10.3846/13926292.2015.1002823

(c) Vilnius Gediminas Technical University, 2015
Publisher: Taylor\&Francis and VGTU

http://www.tandfonline.com/TMMA

ISSN: $1392-6292$

eISSN: $1648-3510$

\title{
Design of Deterministic Model of Signal Transduction between Neuronal Cells*
}

\section{Maryna A. Hliatsevich, Pavel M. Bulai, Taras N. Pitlik, Andrey A. Denisov and Sergey N. Cherenkevich}

\author{
Belarusian State University, Physics Faculty \\ 4 Nezavisimosti av, Minsk, Republic of Belarus \\ E-mail(corresp.): gletsev@bsu . by \\ E-mail: gletsevich.marina@gmail.com
}

Received October 29, 2013; revised December 15, 2014; published online January 15, 2015

\begin{abstract}
Mathematical model describing signal transduction between neurons has been presented using the system of nonlinear ordinary differential equations. The Cauchy problem for the given system has been solved numerically and system parameters were adjusted to match the results of experimental measurements of extracellular postsynaptic potentials in rat hippocampus slices. While fitting model to the experimental data some values of synaptic parameters have been determined.
\end{abstract}

Keywords: deterministic model, synaptic transmission, simulation.

AMS Subject Classification: 34C60; 92B99; 94A12; 92C20.

\section{Introduction}

One of the most acute problems in the life sciences is investigation and reconstruction of the cerebration and communication between neuronal cells. Researches in this area are matter of great importance not only for the fundamental science but also promise significant breakthrough in medicine of brain diseases. According to the data given in [18] direct and indirect costs of brain diseases amount to more than 350 billion euros per year, so brain investigations are also socially and economically important and are directed to raise the quality of life.

To gain a new insight into main principles of brain functioning and neuronal development it is necessary to carry out investigation on different levels of brain organization using complex approaches. One of the particular stages in cerebration is signal transduction between neuronal cells (synaptic transmission (ST)), which lays the molecular basis for information processing in brain. The importance of understanding ST principles resulted in wide spectrum of experimental investigations revealing the main characteristics and mechanisms

\footnotetext{
* This work is supported by Belarusian Republican Foundation for Fundamental Research.
} 
of neuronal interactions. Nevertheless, deep understanding of ST can not be achieved without theoretical confirmation, and huge amount of experimental data requires adequate mathematical models. Therefore nowadays the problem of ST mathematical description is becoming more and more urgent.

To date there is a great number of mathematical models describing different aspects of synaptic transmission. We can distinguish two main approaches to ST modeling. The first approach consists in building mathematical model which does not consider detailed cascade of biochemical reactions and considers synapse as a system with several numbers of states [5]. Usually such models focus on the properties of postsynaptic compartment (i. e. type of neurotransmitter receptor, [5]), they are simulated easily and are very useful in network modelling. On the other hand such simplifications do not provide any information concerning functional significance of biochemical reactants involved in ST. This fact prevents such models from usage for biomedicine purposes. The second approach concentrates on the detailed biophysical model which considers all stages and participants of ST [29]. Such model can take into account heterogeneity of cell compartments, spatial and stochastic factors. Due to their complexity the second type models are usually focused on a particular step of $\mathrm{ST}$, on a particular feature or a type of synapse. Integrating of neuron assembly into network is very complicated for these models.

The main goal of our work is to suggest the generalized biophysical model of synaptic transmission that allows dynamic description of main biochemical participants and on the other hand leaves a possibility of making simplification depending on specific properties of a given synapse. Such model reduction will be based on the qualitative theory of dynamical systems. We offer deterministic model of ST biochemical reactions presented as a system of ordinary differential equations. This model allows to simulate extracellular postsynaptic potentials and may be adjusted for different types of synapses in brain.

While developing the model we paid a specific attention to properties of hippocampal synaptic contacts. Hippocampus formation plays significant role in memory development and spatial perception, so ST in this brain region is of our particular interest. Experimental validation of suggested model is performed for synaptic contacts between CA3 and CA1 areas in rat hippocampus.

\section{Methods}

\subsection{Computation of synaptic current}

Neurons belong to the class of the electroexcitatory cells. As all types of cells they maintain a potential difference across their plasma membrane termed resting potential, but the key feature of the electroexcitatory cells is their possibility to generate propagating wave of depolarization, which is called action potential (AP). Having achieved at the terminal of nerve AP can be transmitted to the following cell due to transformation of electrical signal into chemical one and reverse decoding on the membrane of the next cell. Such signal transformation takes place in the synapses - contacts between neuronal cells, and the type of signal transduction is called synaptic transmission. 
One of the features of synapses is a special morphology and sophisticated organizational system that enables different ways of ST regulation. This regulation has great significance for brain physiology, e. g. memory formation [16]. Synapse is usually divided in three compartments: presynaptic nerve terminal (presynaptic bouton, the place of input of the signal - AP), synaptic cleft (the space between neurons) and postsynaptic terminal. Presynaptic terminal contains synaptic vesicles (SV) that are filled with neurotransmitter (NT) molecules. Each synapse has SVs with only one type of NT. The opposite postsynaptic membrane carries on neurotransmitter receptors for corresponding NT.

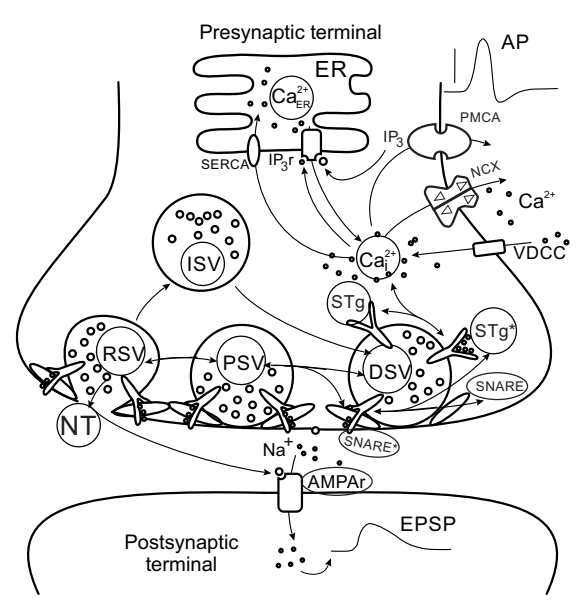

Figure 1. The scheme of synaptic transmission.

Experimental researches carried in the last decades demonstrated that ST of information presents a complicated multistage process involving variety of participants, each of them in its turn can interact with different regulatory pathways in the cell [16]. Schematically we can separate the following main phases of ST represented in Figure 1:

1. AP reaches the axon's terminal and opens the voltage-dependent calcium channels. In the open state these proteins allow calcium ions to move against their concentration gradient into presynaptic bouton resulting a steep increase of cytoplasm calcium ions concentration.

2. In bouton cytoplasm calcium ions bind with special protein sensor. At present the most probable sensor is assumed to be synaptotagmin ( $\mathrm{STg}$ ) protein. It has 5 cites of binding with $\mathrm{Ca}^{2+}$ and attaching of $\mathrm{Ca}^{2+}$ leads to the conformational changes of the protein structure.

3. Bound with $5 \mathrm{Ca}^{2+} \mathrm{STg}$ is enabled to interact with protein complexes that are responsible for docking of SV on the plasma membrane. Such interaction results in fusion of SV membrane and plasma membrane and NT release into the synaptic cleft. 
4. The molecules of neurotransmitter pass through synaptic cleft and reach the postsynaptic membrane where they are recognized by corresponding receptors.

5. One can distinguish two types of neurotransmitter receptors: liganddependent ionic channels (the ionotropic receptors) and G-coupled receptors (the metabotropic receptors). In the first case interaction with neurotransmitter leads to opening of the ionic channels on the neurotransmitter receptors and further synaptic current generation. The changes in the postsynaptic membrane conductivity result in formation of postsynaptic potential wave and its further propagation on the dendrite.

In our suggested model we deal with the first type of neurotransmitter receptors since they have been shown to play a pivotal role in ST in excitatory synapses of hippocampus [16].

\subsubsection{Calcium concentration changes in presynaptic terminal}

In our suggested model we have chosen single rectangle current impulse applied on the presynaptic membrane as an input signal. This impulse leads to generation of AP which we describe with the help of Hodgkin-Huxley-like model equations [12]. The changes in the membrane potential result in $\mathrm{Ca}^{2+}$ influx into cytoplasm involving two major mechanisms.

\section{Regulation of $\mathrm{Ca}^{2+}$ concentration by the proteins on plasma mem-} brane. Neuronal plasma membrane in the zone of synaptic contact includes different types of proteins that regulate $\mathrm{Ca}^{2+}$ concentration in presynaptic bouton and are coupled with exocytosis machinery. The most important proteins are voltage-dependent calcium channel, plasma membrane $\mathrm{Ca}^{2+}$ ATPase (calcium pump PMCA) and $\mathrm{Na}^{+} / \mathrm{Ca}^{2+}$ exchanger (NCX). The first is responsible for the calcium influx into the cell and the last two are responsible for the calcium efflux. Regarding these proteins as has been shown in [8] we can describe the total $\mathrm{Ca}^{2+}$ surface current density through the plasma membrane as a sum of three terms: $j=j_{V D C C}-j_{e}+j_{L}$ where $j_{V D C C}$ stands for the $\mathrm{Ca}^{2+}$ current density through voltage-dependent calcium channels, $j_{e}$ is the $\mathrm{Ca}^{2+}$ current density regulated with PMCA and NCX, $j_{L}$ denotes the leakage current density through the plasma membrane.

Calcium current through voltage-dependent calcium channels. Surface density of calcium current through voltage-dependent calcium channels (VDCC) is defined by difference between membrane potential and calcium reversal potential, and also by the conductance of ionic channels. The latter also depends on membrane potential $j_{V D C C}=\rho_{V} \bar{g}_{V} g_{V}^{2}(V(t))\left(\bar{V}\left(c_{i}\right)-V(t)\right)[8,10]$. Here $\rho_{V}$ stands for the surface density of the calcium channels and is specific for the particular type of cells, $\bar{g}_{V}$ is the maximal conductance for VDCC and is the universal constant, $V(t)$ is the transmembrane potential at given moment $t$, $\bar{V}\left(c_{i}\right)$ is the reversal potential for $C a^{2+}$ depending on intra- and extracellular 
calcium concentration, $g_{V}^{2}$ is continuous value reflecting the part of VDCC in open state. According to the Nernst equation calcium reversal potential is defined as following

$$
\bar{V}\left(c_{i}\right)=\frac{R T}{Z F} \ln \left(\frac{c_{e x t}}{c_{i}}\right) .
$$

Here $R$ stands for universal gas constant, $Z$ is valency of calcium ion and equals to 2, $T$ is absolute temperature, $F$ is Faraday constant, $c_{\text {ext }}$ and $c_{i}$ are extra- and intracellular concentration of calcium ions. Given form of the Nernst equation introduces additional way of the calcium influx regulation provided by $\bar{V}\left(c_{i}\right)$ changes when intracellular concentration of $\mathrm{Ca}^{2+}$ is rising.

The time course of the gate variable $g_{V}$ is usually described with the help of exponential expression [10]:

$$
\frac{d g_{V}}{d t}=\frac{\widehat{g}_{V}-g_{V}}{\tau},
$$

where $\widehat{g}_{V}$ is steady-state value of $g_{V}$ and $\tau$ stands for the time constant. The dependence of $\widehat{g}_{V}$ on the transmembrane potential can be approximated with the Boltzmann expression [8]:

$$
\widehat{g}_{V}=\frac{1}{\left(\exp \left(\left(V_{h}-V\right)\right) / \kappa\right)+1},
$$

where $V_{h}$ is the half-activation potential and $\kappa$ is the steepness factor.

Regulation of calcium flux from bouton into extracellular medium. The main mechanism of excluding calcium ions from cytoplasm of a cell is activity of PMCA and NCX.

NCX is usually assumed to have a low affinity to $\mathrm{Ca}^{2+}$ but for PMCA the opposite situation is observed. Due to this property NCX is responsible for $\mathrm{Ca}^{2+}$ efflux when the $\mathrm{Ca}^{2+}$ concentration rises rapidly and PMCA regulates tiny oscillation of $\mathrm{Ca}^{2+}$ concentration close to its basal level. Molecular structure of these proteins reveals limited activity, which is characteristic for any enzyme. Taking in account this fact we can write a surface density of $\mathrm{Ca}^{2+}$ currents through these structures according to [8]:

$$
j_{e}=\rho_{p} g_{p}\left(c_{i}\right) \bar{I}_{p}+\rho_{x} g_{x}\left(c_{i}\right) \bar{I}_{x} .
$$

Here the index $p$ is connected with PMCA term, the index $x$ is connected with NCX term, $\rho_{p}$ and $\rho_{x}$ are the surface densities of given proteins regarded to be constant and specific to the given type of cells, $\bar{I}_{p}$ and $\bar{I}_{x}$ are the maximal $\mathrm{Ca}^{2+}$ currents through the enzymes, $g_{p}$ and $g_{x}$ are values regulating the level of enzymes activity and are dependent on $\mathrm{Ca}^{2+}$ concentration according to the Hill equation:

$$
g_{p, x}\left(c_{i}\right)=\frac{c_{i}^{n_{p, x}}}{c_{i}^{n_{p, x}}+H_{p, x}^{n_{p, x}}} .
$$

Here $H_{p}$ and $H_{x}$ stand for the concentrations of half-maximum activation of enzymes and $n_{p}$ and $n_{x}$ are the Hill coefficients. 
Plasma membrane of neuron possesses a complicated structure, so we can assume other unknown ways of calcium influx into the cell apart one mentioned above. To take them into account it is necessary to introduce leakage current through membrane. Fit the value of surface density of leakage current to compensate any calcium current at the stationary (without any AP) state $[8,29]$ :

$$
j_{L}=-\left(\bar{j}_{V D C C}-\bar{j}_{e}\right) .
$$

Here $\bar{j}_{V D C C}$ and $\bar{j}_{e}$ represent surface densities of currents through VDCC, PMCA and NCX calculated at initial intracellular $\mathrm{Ca}^{2+}$ concentration and at transmembrane potential that is equal to the resting potential.

Binding of calcium ions with intracellular calcium chelators. The amount of $\mathrm{Ca}^{2+}$ available for calcium sensor which is involved in exocytosis machinery is less than total amount of $\mathrm{Ca}^{2+}$ passed into the cytoplasm. The main reason for this fact is the presence of different calcium chelators. To approach this problem we need to introduce additional term in equation describing $\mathrm{Ca}^{2+}$ concentration changes provided by extracellular medium $\left(d c_{i} / d t\right)_{\text {extr }}$ (in the way given by the authors of [8]):

$$
\left(\frac{d c_{i}}{d t}\right)_{e x t r}=\frac{G}{z F}\left(j_{V D C C}-j_{e}+j_{L}\right)+B\left(c_{i}\right) .
$$

According to the analysis provided in [8] the term $B\left(c_{i}\right)$ can be expressed as the time derivative of concentration of chelator which is bound with $\mathrm{Ca}^{2+}$ (b) in assumption that the time of settling equilibrium state between $\mathrm{Ca}^{2+}$ and chelator is much more shorter than characteristic time of $\mathrm{Ca}^{2+}$ concentration changes:

$$
B=-\frac{d b}{d t}=-\frac{\partial b}{\partial c_{i}}\left(\frac{d c_{i}}{d t}\right)
$$

Such reasoning leads to the expression:

$$
\left(\frac{d c_{i}}{d t}\right)_{e x t r}=\frac{G}{z F}\left(j_{V D C C}-j_{e}+j_{L}\right) \frac{1}{1+T} .
$$

Here the term $T$ depends on the total concentration of chelator in cell $b^{0}$ and ratio of rate constants for $\mathrm{Ca}^{2+}$ dissociation and binding with chelator molecule $K=k^{-} / k^{+}: T=b^{0} K /\left(K+c_{i}\right)^{2}$. $G$ is geometric factor which is equal to the ratio of presynaptic bouton surface to its volume.

Regulation of $\mathrm{Ca}^{2+}$ concentration due to participation of the calcium depot. Extracellular medium is not the only source of $\mathrm{Ca}^{2+}$ ions, this function also belongs to such calcium depots as mitochondria and endoplasmic reticulum (ER). Although calcium oscillations caused by these sources are significantly slower than processes of ST considering of calcium depots can turn out to be significant for investigation of neuron stimulation with series of impulses. It has been shown that slight deviation of $\mathrm{Ca}^{2+}$ concentration from 
basal level can lead to asynchronous (which does not related to AP on the presynaptic membrane) release of NT.

For simplification while building the model we will take in account only the ER because it is ER that plays a crucial role in the regulation of synaptic efficacy, e.g. in the short-term synaptic plasticity [7]. The membrane structure of ER includes calcium ATPase pumping $\mathrm{Ca}^{2+}$ into the depot (SERCA pump) and two kinds of ligand-dependent calcium channels: ryanodine receptors and inositol-3-phosphate receptor (IP3r).

We have chosen the model of calcium efflux from ER devised by Li and Rinzel as a basic one [17]. This model describes changes of $\mathrm{Ca}^{2+}$ concentration caused by the activity of SERCA $\left(J_{\text {ERpump }}\right)$ and IP3r $\left(J_{\text {ERchan }}\right)$ :

$$
\begin{aligned}
J_{\text {ERchan }} & =h v_{1} m_{\infty}^{3} n_{\infty}^{3} q^{3}\left(c_{E R}-c_{i}\right), \quad J_{\text {ERpump }}=\frac{v_{3} c_{i}^{2}}{k_{s}^{2}+c_{i}^{2}}, \\
J_{\text {ERleak }} & =h v_{2}\left(c_{E R}-c_{i}\right) .
\end{aligned}
$$

Here $c_{E R}$ is $\mathrm{Ca}^{2+}$ concentration in ER, $\mathrm{m}_{\infty}^{3}$ is the equilibrium part of IP3rs bound with inositol-3-phosphate at its constant concentration, $n_{\infty}$ is the equilibrium part of the inhibitory IP3r sites bound with $\mathrm{Ca}^{2+}$, which depends on cytoplasmic $\mathrm{Ca}^{2+}$ concentration, $v_{1}$ is the maximal rate of $\mathrm{Ca}^{2+}$ flux through IP3r, $q$ is the part of activated IP3r which is governed by differential equation $d q / d t=\alpha_{q}(1-q)-\beta_{q} q, \alpha_{q}$ and $\beta_{q}$ are the rate constants of IP3r channels opening and closing respectively, $v_{2}$ is the rate constant of $\mathrm{Ca}^{2+}$ leakage from $\mathrm{ER}, v_{3}$ is the SERCA maximal pump rate, $k_{s}$ is SERCA dissociation constant, $h$ is ratio of ER volume to the volume of presynaptic bouton. The quantity of calcium ions in cytoplasm also determines the part of activated IP3r since the rate constant of transition $\beta_{q}$ is proportional to $\mathrm{Ca}^{2+}$ concentration. $J_{\text {ERleak }}$ is the $\mathrm{Ca}^{2+}$ leakage flux from ER to the cytosol.

Therefore considering calcium depots we have derived another unknown variable $c_{E R}$. Li and Rinzel proposed to introduce the total free $\mathrm{Ca}^{2+}$ concentration per cytosolic volume $c_{0}$. Taking this into account, $\mathrm{Ca}^{2+}$ concentration in ER can be described as: $c_{E R}=\left(c_{0}-c_{i}\right) / h$. In [17] it was shown that the changes of $c_{0}$ relate with $\mathrm{Ca}^{2+}$ influx into the cell according to the expression

$$
\frac{d c_{0}}{d t}=\varepsilon\left(\frac{d c_{i}}{d t}\right)_{\text {extr }}
$$

Here $\varepsilon$ is geometric factor equal to the ratio of plasma membrane area to ER membrane area. Therefore considering the both sources of calcium - extracellular medium and calcium depot - we can derive the equation

$$
\frac{d c_{i}}{d t}=\frac{G}{z F}\left(j_{V D C C}-j_{e}+j_{L}\right) \frac{1}{1+T}+\left(J_{\text {ERchan }}-J_{\text {ERpump }}+J_{\text {ERleak }}\right) \frac{1}{1+T} \text {. }
$$

Interaction of calcium ions with calcium sensor protein. Nowadays mechanisms of SV exocytosis attract significant attention of biochemists and electrophysiologists. It has been shown that this process includes sophisticated biochemical cascades involving various participants and possessing different regulation pathways. Since the main goal of our work is generalized description of 
postsynaptic potential as the result of synaptic transmission we will not consider the details of exocytosis regulation mechanisms. According to the results of many researches carried out in last decade the main steps of exocytosis are mediated by SNARE protein complex [16]. The proteins forming this complex are responsible for docking of SV on the bouton membrane but exocytosis can be induced only after interaction of calcium ions with some triggering proteins. Numerous experimental results indicate the fact that the most likely candidate for this function is synaptotagmin (STg) [3]. Activated by $\mathrm{Ca}^{2+} \mathrm{STg}$ is able to induce SNARE-mediated exocytosis.

Structure analysis of STg shows 5 sites of $\mathrm{Ca}^{2+}$ binding. Suppose the processes of interaction of these sites with $\mathrm{Ca}^{2+}$ to be independent from each other and the probability of binding $m$ can be described on the basis of Markov chain's formalism by expression:

$$
\frac{d m}{d t}=\alpha_{m} c_{i}(t)(1-m)-\beta_{m} m .
$$

Here $\alpha_{m}$ and $\beta_{m}$ are the rate constants for binding of $C a^{2+}$ with STg site and for its dissociation correspondingly.

To interact with SNARE complex STg needs to bind $5 \mathrm{Ca}^{2+}$. Therefore if the total amount of STg molecules is $(S T)_{0}$ then the amount of activated synaptotagmin molecules $\left(\mathrm{STg}^{*}\right)$ can be derived as $(S T)_{0} m^{5}$.

Consider the participation of $\mathrm{STg}^{*}$ in exocytosis. Omitting the structure details of interactions between STg* and SNARE complex we represent them by the following chemical reaction:

$$
S T g^{*}+S N A R E \underset{k_{2}}{\stackrel{k_{1}}{\rightleftarrows}} S N A R E^{*} .
$$

Here SNARE* denotes the functionally active STg-SNARE complex which is able to induce the membrane fusion and vesicle exocytosis. Taking into account reactions (2.2) the time course of changes in the amount of $\mathrm{STg}^{*}$ can be described as following:

$$
\frac{d S T^{*}}{d t}=J_{S T^{*}}^{+}-J_{S T^{*}}^{-}
$$

Here $S T^{*}$ is the amount of $\mathrm{STg}^{*}, J_{S T^{*}}^{+}$is the rate of $\mathrm{STg}^{*}$ formation and $J_{S T^{*}}^{-}$ is the rate of its deactivation. Regarding the process of STg cites binding with calcium ions we will derive the expression:

$$
J_{S T^{*}}^{+}=\frac{d}{d t}\left((S T)_{0} \cdot m^{5}\right)=5(S T)_{0} \cdot m^{4} \frac{d m}{d t} .
$$

While applying the law of mass action for the reaction (2.2) we derive the expression for the rate of $\mathrm{STg}^{*}$ deactivation and additional term in the rate of $\mathrm{STg}^{*}$ formation:

$$
J_{S T^{*}}^{-}=k_{1} S_{n} S T^{*}, \quad J_{S T^{*}}^{+}=k_{2} S_{n}^{*} .
$$

Here $S_{n}$ and $S_{n}^{*}$ denote the amount of SNARE and SNARE* complexes correspondingly. The resulting expression looks as:

$$
\frac{d S T^{*}}{d t}=5(S T)_{0} \cdot m^{4} \frac{d m}{d t}+k_{2} S_{n}^{*}-k_{1} S_{n} S T^{*} .
$$


Synaptic vesicles exocytosis. The next important stage of signal transduction in the synapse is fusion of synaptic vesicle membrane and presynaptic bouton membrane and following NT release into synaptic cleft. This process is regulated by various mechanisms and SNARE complex formation and its interaction with STg are assumed to play the major role. It has been shown that not every SV is placed on plasma membrane and ready for exocytosis [28]. According to the scheme proposed in [3] binding of STg with $\mathrm{Ca}^{2+}$ triggers conformational changes of this protein and its partial penetration in specific membrane regions that leads to drawing together membranes of the vesicle and that of the bouton. In parallel STg* drives full SNARE assembly that corresponds to formation of SNARE* in our model. Taking into account these facts consider presynaptic bouton. It contains $N$ synaptic vesicles that one could divide into four pools: docked at the plasma membrane (weak interaction with plasma membrane mediated by non-activated SNARE complex and some others proteins, DSV in Figure 1), primed (prepared for neurotransmitter release by SNARE* complex, PSV in Figure 1), released (vesicle is open into synaptic cleft, RSV in Figure 1) and inactivated (vesicle is involved in process of recycling, ISV in Figure 1). $D, P, R$ and $I$ denote the fractions of $N$ for corresponding vesicle types and we consider the transitions between these pools of vesicles. We assume that there are $n \mathrm{STg}$ molecules on each docked vesicle. We will take into account only these molecules since only this part of STg can mediate the exocytosis and other STg molecules can be considered as calcium chelator described above. Therefore, the total number of STg molecules in bouton is derived as $n D N$. Furthermore, $l$ activated SNARE complexes on the docked vesicle are needed for exocytosis initiation [3], and transitions between states of vesicle could be represented by the scheme in Figure 2 and equation $I=1-D-R-P$.

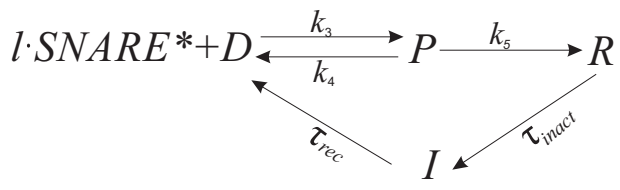

Figure 2. The scheme of SV transitions between pools.

The law of mass action applied to given scheme will set the system of following equations for the scheme description:

$$
\begin{aligned}
& \frac{d D}{d t}=-k_{3} D\left(S_{n}^{*}\right)^{l}+k_{4} P+\frac{I}{\tau_{r e c}}, \\
& \frac{d P}{d t}=k_{3} D\left(S_{n}^{*}\right)^{l}-\left(k_{4}+k_{5}\right) P, \\
& \frac{d R}{d t}=k_{5} P-\frac{R}{\tau_{\text {inact }}} .
\end{aligned}
$$

Here $\tau_{\text {rec }}$ and $\tau_{\text {inact }}$ are the time constants for synaptic vesicle recovery and inactivation, as was introduced in [30]. The process of synaptic vesicle recovery is transition from $I$ pool into $D$ pool, and inactivation is process of converting 
$R$ vesicle into $I$ vesicle. $k_{3}, k_{4}$ and $k_{5}$ are the rate constants for the transitions illustrated in Figure 2.

Opening of SV leads to NT (in our case glutamate) release into synaptic cleft and elevation of its concentration. According to [29] the time course of glutamate concentration $\Gamma$ in the cleft can be described with the equation

$$
\frac{d \Gamma}{d t}=n_{v} g_{N T} R-g_{c} \Gamma
$$

where $n_{v}$ is the number of docked vesicle, $g_{N T}$ is apparent concentration of glutamate in synaptic vesicle and $g_{c}$ is the rate constant of glutamate clearance due to diffusion from synapse side and reversal uptake by presynaptic neuron.

Neurotransmitter receptors. Proteins responsible for NT recognition NT receptors - are placed on the opposite postsynaptic membrane. The type of recognized NT and molecular structure control the various electrophysiological characteristics such as selectivity, maximal ionic conductance, time constants and also determine the antagonists of receptor. The most widespread excitatory synapses in hippocampus involve receptors of $\alpha$-amino-3-hydroxy-5methyl-4-isoxazolepropionic acid (AMPA) and kainate and inhibitory synapses recruit receptors of $\gamma$-aminobutyric acid (GABA). We will deal with excitatory synapses.

AMPA receptor (AMPAr) belongs to the class of ionotropic receptors, in other words, ligand binding leads to its opening and ion flux through the AMPAr into the terminal. This process involves six states of AMPAr which include different types of closed and desensitized states and one open state. Transitions between these states are assumed to represent Markov process. It has been shown that opening of AMPAr channels can be adequately described if one considers only two states - open state and closed one [5]. The probability of transition from open state to the closed one is proportional to NT concentration in the synaptic cleft:

$$
C \underset{\beta}{\stackrel{k_{6}}{\rightleftarrows}} O
$$

Taking into account this Markov chain we can describe the time course of single channel open probability $O$ with the help of equation:

$$
\frac{d O}{d t}=k_{6} \Gamma(1-O)-\beta O \text {. }
$$

Here $k_{6}$ and $\beta$ are the rate constants of AMPAr's channels opening and closing correspondingly.

The current mediated by ionic channel of AMPAr, $I_{s y n}$, is the product of maximal channel conductance $g_{A M P A}$, single channel open probability $O$ and potential difference $V-V_{A M P A}$ where $V$ is transmembrane potential and $V_{A M P A}$ is reversal potential of AMPAr channel [5]:

$$
I_{\text {syn }}=g_{A M P A}\left(V-V_{A M P A}\right) O .
$$

Current impulse passed through open channels of AMPArs initiates generation of AP on the postsynaptic membrane that we can describe by Hodgkin-Huxleylike equations. 


\subsection{Formation of extracellular postsynaptic potential}

Experimentally ST is investigated with the help of microelectrode technique. The hippocampal slice is one of the most used preparations for this technique and is aimed to the study of the mammalian central nervous system in general and synaptic phenomena in particular. In such experiments stimulating electrode is usually placed on CA3 Schaffer collateral fibers while one or several recording electrodes are placed into the stratum radiatum of area CA1. The recording electrodes register extracellular field potentials that represent the summed responses from a number of neurons in the vicinity of the recording electrode. To have possibility for relevant comparison of simulation and experimental result we have constructed the equivalent scheme of contact between postsynaptic neuron and microelectrode. We do not pose a problem to investigate the peculiarities of signal transduction from the dendrite to the soma, therefore, we will deal with only two neuron compartments - the "generalized" dendrite and the soma. While regarding the connected tip of microelectrode one should take into account the conductance $g_{e l}$ and capacitance $C_{e l}$ of electrical double layer on the tip [22]. The measured current is taken from the resistor with conductance $g_{\text {out }}$. This contact configuration can be described by the equivalent electrical scheme presented in Figure 3.

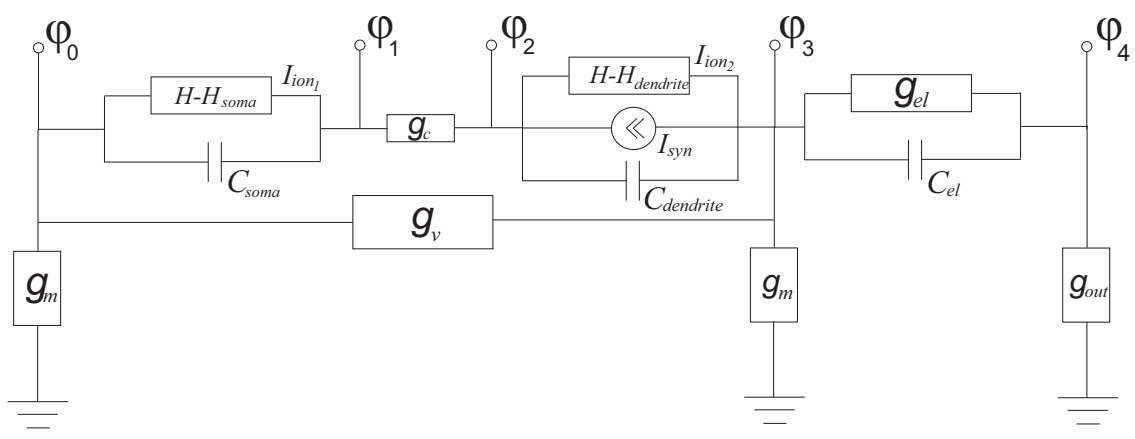

Figure 3. The equivalent electrical scheme of two-compartment neuron.

Here $g_{m}$ represents the leakage conductance, $g_{v}$ is conductance of the extracellular medium, $g_{c}$ is the conductance between cell compartments.

Applying Kirchhoff's laws and Hodgkin-Huxley-like model to presented circuit we obtain the next set of equations:

$$
\begin{aligned}
& C_{\text {soma }} \frac{d\left(\varphi_{1}-\varphi_{0}\right)}{d t}+I_{\text {ion } 1}=-\left(\varphi_{3} g_{m}+\varphi_{4} g_{\text {out }}\right)+\left(\varphi_{0}-\varphi_{3}\right) g_{v}, \\
& \left(\varphi_{1}-\varphi_{2}\right) g_{c}+\left(\varphi_{0}-\varphi_{3}\right) g_{v}=-\varphi_{0} g_{m}, \\
& -\varphi_{0} g_{m}=\varphi_{3} g_{m}+\varphi_{4} g_{\text {out }}, \\
& C_{\text {dendrite }} \frac{d\left(\varphi_{2}-\varphi_{3}\right)}{d t}+I_{\text {ion } 2}+I_{\text {syn }}=\left(\varphi_{3} g_{m}+\varphi_{4} g_{\text {out }}\right)-\left(\varphi_{0}-\varphi_{3}\right) g_{v}, \\
& C_{e l} \frac{d\left(\varphi_{3}-\varphi_{4}\right)}{d t}+\left(\varphi_{3}-\varphi_{4}\right) g_{\text {el }}=\varphi_{4} g_{\text {out }} .
\end{aligned}
$$


$I_{\text {syn }}$ is the synaptic current given by the formula $(2.3)$ and $I_{i o n}$ is the sum of sodium, potassium, and leakage currents. Dealing with hippocampal slices of rats we consider various types of ionic channels for each type of current. We take into account currents and dependence of conductance on voltage described in $[25]$ :

$$
\begin{aligned}
I_{i o n}= & g_{L}\left(V_{m}-V_{L}\right)+\bar{g}_{N a} m^{3} h\left(V_{m}-V_{N a}\right)+\bar{g}_{N a P} w\left(V_{m}-V_{N a}\right) \\
& +\bar{g}_{V} u\left(V_{m}-V_{K}\right)+\bar{g}_{A} a b\left(V_{m}-V_{K}\right)+\bar{g}_{D R} n^{4}\left(V_{m}-V_{K}\right) .
\end{aligned}
$$

Here $V_{m}$ is the transmembrane potential, $V_{L}, V_{N a}, V_{K}$ are the reversal potentials of leakage current, sodium and potassium ionic channels correspondingly, $g_{L}, \bar{g}_{N a}, \bar{g}_{N a P}, \bar{g}_{V}, \bar{g}_{A}, \bar{g}_{D R}$ are the maximal conductances of corresponding ionic channel, $m, h, w, u, a, b, n$ are the gate variables. Each gate variable $x$ is governed by the equation for two-state Markov process:

$$
\frac{d x}{d t}=\alpha_{x}(1-x)-\beta_{x}
$$

Rate functions $\alpha_{x}$ and $\beta_{x}$ are taken as given in [25]. On the basis of equations (2.4) we have derived the expression for $\varphi_{4}$ :

$$
\varphi_{4}=-\frac{g_{\text {out }}\left(\left(\varphi_{0}-\varphi_{1}+\varphi_{2}+\varphi_{3}\right) g_{c}+\varphi_{3}\left(g_{m}+2 g_{v}\right)\right)}{g_{\text {out }}\left(g_{c}+g_{m}+g_{v}\right)} .
$$

The resulting signal is proportional to the $\varphi_{4}$ and coefficient of proportionality depends on the microelectrode characteristics as well as the experimental conditions (the thickness of brain slice, the depth of microelectrode penetration and some others). These conditions are varied in different experiments and can not be controlled precisely. Therefore, we aim to simulate only the form of experimental signal and further while comparing experimental and simulation results take them both scaled by the minimum value.

\section{Results and Discussion}

Mediated by neurotransmitter receptors inward synaptic current initiates postsynaptic potential (PSP) on the dendrite membrane and its propagation to the soma of neuron. Generation of AP on the soma is the result of spatial summation of PSPs that are developed on the ensemble of synapses. PSP of single synapse might be not enough for AP generation on the soma, and the number of excited synapses with a glance of their synaptic weights should exceed some threshold value to initiate the somatic AP. In our model we take into account different levels of synaptic excitation by manipulating with parameter $g_{A M P A}$, which reflects the maximal conductance of AMPArs on the postsynaptic membrane and is a product of AMPAr surface density, the area of the active zone and maximal conductance of single receptor. The last parameter is fixed, while the increase of the first and the second ones may reflect the increase of excited synapses number. According to the investigations presented in [4,19], electrical signals from dendrites are summarized and temporal summation at CA1 pyramidal somata does not depend on the location of synaptic input. Because 

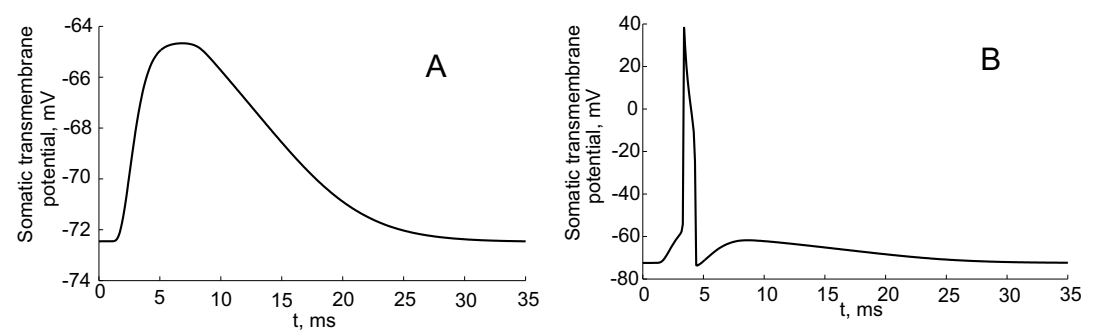

Figure 4. Simulation of the somatic transmembrane potential $\left(\varphi_{1}-\varphi_{0}\right) . A-g_{A M P A}=40$ $\mathrm{mS}, g_{N T}=60 \mu \mathrm{M}, \mathrm{B}-g_{A M P A}=180 \mathrm{mS}, g_{N T}=60 \mu \mathrm{M}$.
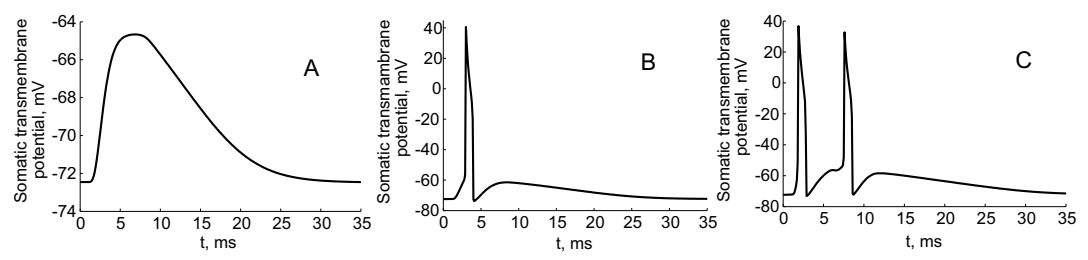

Figure 5. Simulation of the somatic transmembrane potential $\left(\varphi_{1}-\varphi_{0}\right)$. A $g_{A M P A}=40 \mathrm{mS}, g_{N T}=60 \mu \mathrm{M}, \mathrm{B}-g_{A M P A}=25 \mathrm{mS}, g_{N T}=450 \mu \mathrm{M}, \mathrm{C}-g_{A M P A}=180$ $\mathrm{mS}, g_{N T}=450 \mu \mathrm{M}$.

of omitting propagation processes on the dendrites we suggest synaptic current to be proportional to the number of the excited synapses. We can reformulate given postulation by statement that rise of $g_{A M P A}$ over definite value should lead to the appearance of the somatic AP.

We simulated devised model with various sets of parameters to establish the main characteristics and check whether they are in accordance with defined synapses properties. While changing the parameter $g_{A M P A}$ we have observed formation of AP on the soma (Figure 4). This result is in accordance with the assumption that $g_{A M P A}$ is proportional to the number of excited dendrites. Another parameter that should govern the effectiveness of synaptic transmission is NT amount in the synaptic vesicle. This value corresponds to parameter $g_{N T}$ in our model. Indeed, increase in $g_{N T}$ leads to generation of AP on the soma even when $g_{A M P A}$ is lowered (Figure 5).

For further verification of proposed model we compare the experimental results and results of model simulation. Microeletrode technique allows to choose the mode of stimulation that does not lead to the generation of somatic AP (subthreshold stimulation). In this case the extracellular field potentials in the dendritic region are mediated mainly by the synaptic current rather than membrane currents through the potassium and sodium ionic channels. We have chosen this mode of stimulation since the synaptic currents are of our particular interest. The series of extracellular postsynaptic potentials (EPSP) was measured and we calculated the average through the twenty EPSP. Further we adjusted the model parameters to get the optimum coincidence between the forms of measured EPSP and simulated one.

The constructed model of ST includes a lot of parameters (rate constants 
of biochemical reactions, ionic channels densities and others). The majority of them can be taken from biochemical experiments, but some parameters are measured for another types of synapses and need to be made more exact for the regarded synapses. Another set of parameters can not be measured directly and should be derived by model adjusting to the experimental results (hidden parameters). The quantity of parameters that can be determined in the process of model adjusting leads to the unsatisfactory long execution time of the standard fitting procedures that are provided in the mathematical packages (for example, the prediction-error minimization algorithm in Matlab (B).To avoid this problem we have chosen the relevant number of parameters to be adjusted (eight parameters) and regard them in short intervals that have been estimated on the bases of biochemical experimental results provided in the literature. The chosen parameters and their intervals are shown in Table 1.

Table 1. The list of adjusted parameters involved in model.

\begin{tabular}{llll}
\hline Symbol & Minimal value & Maximum value & Step \\
\hline$G$ & $10 \mu \mathrm{m}^{-1}$ & $16 \mu \mathrm{m}^{-1}$ & $2 \mu \mathrm{m}^{-1}$ \\
$\bar{g}_{V}$ & $7 \mathrm{pS}$ & $9 \mathrm{pS}$ & $1 \mathrm{pS}$ \\
$k_{1}$ & $1.1 \cdot 10^{-3} \mathrm{~ms}^{-1}$ & $2 \cdot 10^{-3} \mathrm{~ms}^{-1}$ & $0.3 \cdot 10^{-3} \mathrm{~ms}^{-1}$ \\
$k_{3}$ & $0.5 \cdot 10^{-6} \mathrm{~ms}^{-1}$ & $5 \cdot 10^{-5} \mathrm{~ms}^{-1}$ & $0.2 \cdot 10^{-5} \mathrm{~ms}^{-1}$ \\
$\tau_{\text {inact }}$ & $2.4 \mathrm{~ms}$ & $3.3 \mathrm{~ms}$ & $0.3 \mathrm{~ms}$ \\
$g_{c}$ & $30 \mathrm{~ms}^{-1}$ & $45 \mathrm{~ms}^{-1}$ & $5 \mathrm{~ms}^{-1}$ \\
$k_{6}$ & $1.5 \mathrm{mM}^{-1} \mathrm{~ms}^{-1}$ & $1.9 \mathrm{mM}^{-1} \mathrm{~ms}^{-1}$ & $0.2 \mathrm{mM}^{-1} \mathrm{~ms}^{-1}$ \\
$\beta$ & $0.4 \mathrm{~ms}^{-1}$ & $0.6 \mathrm{~ms}^{-1}$ & $0.05 \mathrm{~ms}^{-1}$ \\
\hline
\end{tabular}

For each varied parameter the relevant step has been chosen in the 8dimension space and for each set of parameters the relative standard deviation of model from experiment has been calculated. We have chosen the set of parameteres that corresponds to the minimum of relative standard deviation. All obtained parameter values are relevant from the biochemistry point of view. Parameters that were fixed in the model can be found in the corresponding articles (Table 2).

The comparison of experimental and model simulation results is presented in Figure 6.

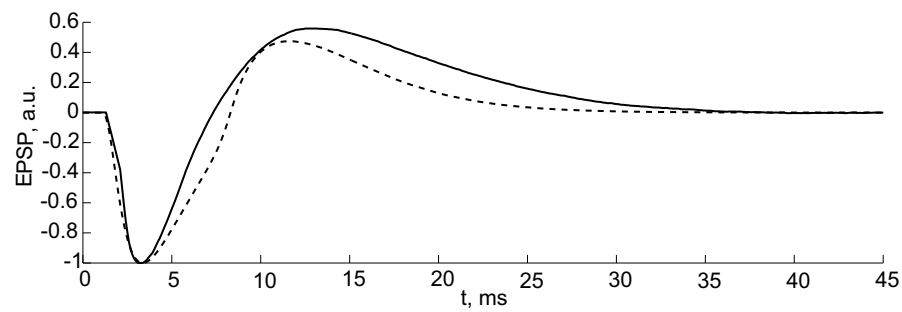

Figure 6. Comparison of experimental results $(-)$ and model simulation $\left(--_{-}--_{-}\right)$. EPSPs from rat hippocampus slices were recorded as described in [27]. 
Table 2. The list of values of parameters involved in the model

\begin{tabular}{|c|c|c|c|}
\hline Symbol & Description & Value & Ref. \\
\hline$\tau$ & $g_{V}$ time constant & $1.0 \mathrm{~ms}$ & {$[9]$} \\
\hline$V_{h}$ & half-activation VDCC voltage & $-9.9 \mathrm{mV}$ & {$[13]$} \\
\hline$\kappa$ & steepness factor $\widehat{g}_{V}$ & $9.4 \mathrm{mV}$ & {$[13]$} \\
\hline$G$ & the bouton surface to its volume ratio & $12 \mu m^{-1}$ & adjusted \\
\hline$\rho_{V}$ & surface density of VDCC & $3.1 \mu m^{-2}$ & {$[8]$} \\
\hline $\bar{g}_{V}$ & $\begin{array}{l}\text { apparent maximal conductance of single } \\
\text { VDCC }\end{array}$ & $8 \mathrm{pS}$ & adjusted \\
\hline$c_{\text {ext }}$ & extracellular $\mathrm{Ca}^{2+}$ concentration & $1.5 \mathrm{mM}$ & {$[11]$} \\
\hline$\rho_{p}$ & surface density of PMCA & $9.2 \cdot 10^{3} \mu m^{-2}$ & {$[8]$} \\
\hline$\rho_{x}$ & surface density of NCX & $3.04 \cdot 10^{2} \mu m^{-2}$ & {$[14]$} \\
\hline $\bar{I}_{p}$ & maximal activity rate of PMCA & $2.7 \cdot 10^{-21} \mathrm{C} / \mathrm{ms}$ & {$[6]$} \\
\hline $\bar{I}_{x}$ & maximal activity rate of $\mathrm{NCX}$ & $4.8 \cdot 10^{-19} \mathrm{C} / \mathrm{ms}$ & {$[14]$} \\
\hline$n_{p}$ & Hill coefficient for PMCA & 2 & {$[6]$} \\
\hline$n_{x}$ & Hill coefficient for NCX & 1 & {$[8]$} \\
\hline$H_{p}$ & half-activation concentration for PMCA & $0.09 \mu M$ & {$[6]$} \\
\hline$H_{x}$ & half-activation concentration for NCX & $1.8 \mu M$ & {$[1]$} \\
\hline$K$ & $k^{-} / k^{+}$for calcuim binding with chelator & $0.5 \mu M$ & {$[15]$} \\
\hline$h$ & ER volume to volume of bouton ratio & 0.185 & {$[26]$} \\
\hline$v_{1}$ & $\begin{array}{l}\text { maximal rate of calcium influx through IP3 } \\
\text { receptor }\end{array}$ & $0.06 \mathrm{~ms}^{-1}$ & {$[17]$} \\
\hline$v_{2}$ & $\mathrm{RT}^{*}$ of $C a^{2+}$ leakage from ER & $0.2 \cdot 10^{-4} \mathrm{~ms}^{-1}$ & {$[17]$} \\
\hline$v_{3}$ & SERCA maximal pump rate & $0.6 \cdot 10^{-3} \mu M m s^{-1}$ & {$[17]$} \\
\hline$k_{s}$ & SERCA dissociation constant & $0.18 \mu M$ & {$[17]$} \\
\hline$\varepsilon$ & $\begin{array}{l}\text { the ratio of plasma membrane area to ER } \\
\text { membrane area }\end{array}$ & 0.01 & {$[17]$} \\
\hline$\alpha_{m}$ & $\mathrm{Ca}^{2+}$ association $\mathrm{RT}^{*}$ for $\mathrm{STg}$ cite & $4 \mu M^{-1} m s^{-1}$ & {$[20]$} \\
\hline$\beta_{m}$ & $\mathrm{Ca}^{2+}$ dissociation $\mathrm{RT}^{*}$ for STg cite & $2.1 \mathrm{~ms}^{-1}$ & {$[20]$} \\
\hline$k_{1}$ & forward $\mathrm{RT}^{*}$ for SNARE* formation & $1.1 \cdot 10^{-3} \mathrm{~ms}^{-1}$ & adjusted \\
\hline$k_{2}$ & backward RT* for SNARE* formation & $8 m s^{-1}$ & {$[2]$} \\
\hline$k_{3}$ & forward $\mathrm{RT}^{*}$ for $D \rightarrow P$ transition & $5.0 \cdot 10^{-6} \mathrm{~ms}^{-1}$ & adjusted \\
\hline$k_{4}$ & backward $\mathrm{RT}^{*}$ for $D \rightarrow P$ transition & $1 m s^{-1}$ & {$[30]$} \\
\hline$k_{5}$ & forward $\mathrm{RT}^{*}$ for $P \rightarrow R$ transition & $10^{3} \mathrm{~ms}^{-1}$ & {$[30]$} \\
\hline$\tau_{\text {rec }}$ & time constant for recovery of SV & $800 \mathrm{~ms}$ & {$[30]$} \\
\hline$\tau_{\text {inact }}$ & time constant for inactivation of SV & $3.3 \mathrm{~ms}$ & adjusted \\
\hline$n_{v}$ & number of docked vesicle & 8 & {$[24]$} \\
\hline$g_{N T}$ & apparent glutamate concentration in vesicle & $60 m M$ & {$[21]$} \\
\hline$g_{c}$ & rate constant of glutamate clearance & $45 \mathrm{~ms}^{-1}$ & adjusted \\
\hline$k_{6}$ & RT $^{*}$ of AMPAr's channel opening & $1.7 m M^{-1} m s^{-1}$ & adjusted \\
\hline$\beta$ & RT* of AMPAr's channel closing & $0.45 \mathrm{~ms}^{-1}$ & adjusted \\
\hline$V_{A M P A}$ & AMPA receptor reversal potential & $0 \mathrm{mV}$ & {$[5]$} \\
\hline$g_{m}$ & conductance of leakage & $2.1 \cdot 10^{-5} \mathrm{~S}$ & {$[23]$} \\
\hline$g_{c}$ & conductance between cell compartments & $2.5 \cdot 10^{-5} \mathrm{~S}$ & {$[23]$} \\
\hline$g_{\text {out }}$ & conductance of the microelectrode tip & $7.2 \cdot 10^{-3} \mathrm{~S}$ & {$[22]$} \\
\hline$g_{e l}$ & conductance of the electrical double layer & $1 \cdot 10^{-10} \mathrm{~S}$ & {$[22]$} \\
\hline$g_{v}$ & conductance of the extracellular medium & $2.5 \cdot 10^{-4} \mathrm{~S}$ & {$[23]$} \\
\hline$C_{\text {soma }}$ & capacity of soma & $10^{-5} \mu F$ & {$[25]$} \\
\hline$C_{\text {dendrite }}$ & capacity of dendrite & $2.5 \cdot 10^{-4} \mu F$ & {$[25]$} \\
\hline$C_{e l}$ & capacity of the electrical double layer & $0.1 \mathrm{nF}$ & {$[22]$} \\
\hline
\end{tabular}

* RT - rate constant 


\section{Conclusions}

We have presented deterministic model of ST as the system of nonlinear ordinary differential equations. Such approach enabled us to determine the set of physiological parameters indirectly. In particular when approximating the results of EPSP microelectrode measurements with simulations given by suggested model we found the values of following parameters characteristic for synapses of rat hippocampus: the bouton surface to volume ratio, apparent maximal conductance of single VDCC, forward rate constant for SNARE* formation, forward rate constant for $D \rightarrow P$ transition, time constant for inactivation of $\mathrm{SV}$, rate constant of glutamate clearance from synaptic cleft, rate constants of AMPAr's channels opening and closing (see Table 2). The values of the bouton surface to volume ratio, apparent maximal conductance of single VDCC, time constant for inactivation of SV, rate constant of glutamate clearance from synaptic cleft, rate constants of AMPAr's channels opening and closing may be found in literature for another types of neurons, but other parameters have been determined for the first time. Therefore one can conclude that developed model could be used as a tool for revealing of values that could not be found experimentally.

Presented way of model developing makes it possible to investigate numerically particular steps of ST and model also allows us to analyze the influence of the definite parameters on the synaptic efficiency as it was shown for parameters $g_{A M P A}$ and $g_{N T}$.

It should be noted that suggested model of synaptic transmission does not pretend to describe this process thoroughly. First of all we consider all variables in the model to be continuous, although some of them are intrinsically discrete such as number of synaptic vesicles or synaptotagmin molecules. Therefore this model can be improved by introduction some probability factors for description of vesicle exocytosis. Nevertheless simplifications made in our model allow us to construct easily simulated and comprehensible mathematical description of such sophisticated process as synaptic transmission. Future investigations include experimental measurements with usage of different stimulation protocols and other various conditions for complete validation of model.

Suggested model will be useful for complex neural network building, investigation of mechanisms governing neurodegenerative diseases and for development and screening of pharmaceutical drug candidates.

\section{References}

[1] M.P. Blaustein and W.J. Lederer. Sodium/calcium exchange: Its physiological implications. Physiological Reviews, 79(3):763-854, 1999.

[2] J.H. Bollmann, B. Sakmann and J.G. Borst. Calcium sensitivity of glutamate release in a calyx-type terminal. Science, 289(5481):953-957, 2000. http://dx.doi.org/10.1126/science.289.5481.953.

[3] E.R. Chapman. Synaptotagmin: A Ca2+ sensor that triggers exocytosis? Nat. Rev. Mol. Cell. Biol., 3(7):498-508, 2002. 
[4] A.E. Desjardins, Y. Li, S. Reinker, R.M. Miura and R.S. Neuman. The influences of Ih on temporal summation in hippocampal CA1 pyramidal neurons: A modeling study. J. Comput. Neurosci., 15(2):131-142, 2003. http://dx.doi.org/10.1023/A:1025881007453.

[5] A. Destexhe, Z.F. Mainen and T.J. Sejnowski. Synthesis of models for excitable membranes, synaptic transmission and neuromodulation using a common kinetic formalism. J. Comput. Neurosci., 1(3):195-230, 1994. http://dx.doi.org/10.1007/BF00961734.

[6] N.L. Elwess, A.G. Filoteo, A. Enyedi and J.T. Penniston. Plasma membrane $\mathrm{Ca} 2+$ pump isoforms $2 \mathrm{a}$ and $2 \mathrm{~b}$ are unusually responsive to calmodulin and Ca2+. J. Biological Chemistry, 272(29):17981-17986, 1997.

[7] N.J. Emptage, C.A. Reid and A. Fine. Calcium stores in hippocampal synaptic boutons mediate short-term plasticity, store-operated ca2+ entry, and spontaneous transmitter release. Neuron, 29(1):197-208, 2001. http://dx.doi.org/10.1016/S0896-6273(01)00190-8.

[8] F. Erler, M. Meyer-Hermann and G. Soff. A quantitative model for presynaptic free $\mathrm{Ca} 2+$ dynamics during different stimulation protocols. Neurocomputing, 61(1):169-191, 2004. http://dx.doi.org/10.1016/j.neucom.2003.11.002.

[9] R.E. Fisher, R. Gray and D. Johnston. Properties and distribution of single voltage-gated calcium channels in adult hippocampal neurons. J. Neurophysiology, 64(1):91-104, 1990.

[10] J. Garcia-Colunga, R. Valdiosera and U. Garcia. P-type Ca2+ current in crayfish peptidergic neurones. J. Experimental Biology, 202(4):429-440, 1999.

[11] B. Hille. Ion Channels of Excitable Membranes. University of Washingthon, 2001.

[12] A.L. Hodgkin and A.F. Huxley. A quantitative description of membrane current and its application to conduction and excitation in nerve. J. Physiology, 117(4):500-544, 1952.

[13] T. Ishikawa, M. Kaneko, H.-S. Shin and T. Takahashi. Presynaptic N-type and $\mathrm{P} / \mathrm{Q}$-type Ca2+ channels mediating synaptic transmission at the calyx of Held of mice. J. Physiology, 568(1):199-209, 2005.

http://dx.doi.org/10.1113/jphysiol.2005.089912.

[14] M. Juhaszova, P. Church, M.P. Blaustein and E.F. Stanley. Location of calcium transporters at presynaptic terminals. Trends Neurosci., 12(3):839-846, 2000.

[15] H.J. Koester and B. Sakmann. Calcium dynamics associated with action potentials in single nerve terminals of pyramidal cells in layer $2 / 3$ of the young rat neocortex. J. Physiology, 529(3):325-646, 2000. http://dx.doi.org/10.1111/j.1469-7793.2000.00625.x.

[16] D.M. Kullmann. The hippocampus book. In R.M.P. Andersen, D. Amaral, T. Bliss and J. O'Keefe(Eds.), Synaptic Function, pp. 203-242. Oxford University Press, Inc., 2007.

[17] Y. Li and J. Rinzel. Equations for InsP3 receptor-mediated [Ca2+]i oscillations derived from a detailed kinetic model: A Hodgkin-Huxley like formalism. $J$. Theoret. Biol., 166(4):461-473, 1994.

[18] C. Lüscher and J.T. Isaac. The synapse: center stage for many brain diseases. J. Physiology, 587(4):727-729, 2009. http://dx.doi.org/10.1113/jphysiol.2008.167742. 
[19] J.C. Magee. Dendritic lh normalizes temporal summation in hippocampal CA1 neurons. Nat. Neurosci., 2(6):508-514, 2002. http://dx.doi.org/10.1038/9158.

[20] O. Millet, P. Bernado, J. Garcia, J. Rizo and M. Pons. Nmr measurement of the off rate from the first calcium-binding site of the synaptotagmin I C2A domain. FEBS Letters, 516(1):93-96, 2002.

http://dx.doi.org/10.1016/S0014-5793(02)02508-5.

[21] V. Montana, E.B. Malarkey, C. Verderio, M. Matteoli and V. Parpura. Vesicular transmitter release from astrocytes. Glia, 54(7):700-715, 2006.

http://dx.doi.org/10.1002/glia.20367.

[22] D.A. Robinson. The electrical properties of metal electrodes. Proceedings of the IEEE, 59(6):1065-1071, 1968. http://dx.doi.org/10.1109/PROC.1968.6458.

[23] A.R. Sargsyan, C. Papatheodoropoulos and G.K. Kostopoulos. Modeling of evoked field potentials in hippocampal CA1 area describes their dependence on NMDA and GABA receptors. J. Neurosci. Methods, 104(2):143-153, 2001. http://dx.doi.org/10.1016/S0165-0270(00)00334-4.

[24] T. Schikorski and C.F. Stevens. Quantitative ultrastructural analysis of hippocampal excitatory synapses. J. Neurosci., 17(15):5858-5867, 1997.

[25] J. Shuai, M. Bikson, P.J. Hahn, J. Lian and D.M. Durand. Ionic mechanisms underlying spontaneous CA1 neuronal firing in Ca2+-free solution. Biophys. J., 84:2099-2111, 2004. http://dx.doi.org/10.1016/S0006-3495(03)75017-6.

[26] J.-W. Shuai and P. Jung. Stochastic properties of Ca2+ release of inositol 1,4,5trisphosphate receptor clusters. Biophys. J., 83(1):87-97, 2002.

http://dx.doi.org/10.1016/S0006-3495(02)75151-5.

[27] E. Stone, K. Hoffman and M. Kavanaugh. Identifying neurotransmitter spill-over in hippocampal field recordings. Math. Biosci., 240(2):169-186, 2012. http://dx.doi.org/10.1016/j.mbs.2012.07.004.

[28] T.C. Südhof. The synaptic vesicle cycle. Annu. Rev. Neurosci., 27(1):509-547, 2004. http://dx.doi.org/10.1146/annurev.neuro.26.041002.131412.

[29] S.G. Tewari and K.K. Majumdar. A mathematical model of the tripartite synapse: astrocyte-induced synaptic plasticity. J. Biological Physics, 94:465496, 2012. http://dx.doi.org/10.1007/s10867-012-9267-7.

[30] M.V. Tsodyks and H. Markram. The neural code between neocortical pyramidal neurons depends on neurotransmitter release probability. Proc. Natl. Acad. Sci., 94(2):719-723, 1997. http://dx.doi.org/10.1073/pnas.94.2.719. 